\title{
Plasmonic Interaction between Overlapping Nanowires
}

\author{
Dang Yuan Lei, Alexandre Aubry,* Yu Luo, Stefan A. Maier, and John B. Pendry \\ The Blackett Laboratory, Department of Physics, Imperial College London, London SW7 2AZ, United Kingdom
}

M etallic nanoparticle dimers have attracted considerable research interests because their plasmonic properties can be controlled by tuning the intraparticle or interparticle distance. ${ }^{1-5}$ On the one hand, the plasmon hybridization model, an electromagnetic analogue of molecular orbital theory, has been proposed and used to explain qualitatively and intuitively the plasmon resonance splitting in metal nanoshells and spherical metallic nanoparticle dimers. ${ }^{2,4}$ On the other hand, some experiments have shown that flat nanodisk dimer arrays exhibit an abrupt transition and a splitting of the plasmon resonance energy when varying the interparticle separation within the pairs from dielectric proximity (nontouching dimers) to conductive contact (overlapping dimers). ${ }^{3}$ Subsequent numerical studies performed for overlapping spherical dimers have confirmed these experimental observations. ${ }^{5}$ However, the transition between these two regimes has remained unclear because of the singularity arising in the limit of touching dimers.

Recently, transformation optics has proven to be an efficient and elegant tool to study analytically the optical response of complex nanostructures with structure singularities. ${ }^{6-11} \mathrm{~A}$ broad-band response and a huge field enhancement have been predicted for kissing nanowires, ${ }^{6,7}$ which might find applications in single-molecule detection, ${ }^{12}$ surface-enhanced Raman scattering (SERS), ${ }^{13}$ and high-harmonic generation. ${ }^{14}$ However, the ultra-broad-band feature displayed by these nanostructures highly relies on their structure singularities, which imposes a severe challenge on the fabrication of these devices. From an experimental point of view, a system of overlap-

www.acsnano.org
ABSTRACT The plasmonic interaction between overlapping nanowires with and without structure singularities is studied analytically and numerically. A conformal transformation approach is adopted to predict analytically the optical response of overlapping nanowires in the quasi-static limit. Surface plasmon excitations are shown to exhibit a lower bound cutoff frequency, which blue-shifts when the overlap distance increases. Between this cutoff and the surface plasmon frequencies, overlapping nanowires are capable of a strong and broad-band harvesting of light. This band gap feature is shown to be robust to radiative losses and to the bluntness of the structure singularities. Hence, the light harvesting performance of overlapping nanowires would not be damaged by nanofabrication imperfections. These remarkable features might be beneficial to the realization of plasmonic band gap filters.

KEYWORDS: plasmonics - transformation optics - broad-band light harvesting · band gap filters $\cdot$ nanofocusing $\cdot$ overlapping nanowires

ping nanowires without any structural singularity is more realistic. Such a geometry could, for example, consist of dimers touching in more than one point fabricated via annealing. The bluntness of singularities in such plasmonic nanostructures and its impact on their optical response have not been addressed so far. ${ }^{6-9}$ Recently, Bora et al. have successfully fabricated closely packed parallel gold nanowire arrays with a gap distance of a few tens of nanometers as tunable plasmon resonant cavities. ${ }^{15}$ This work might design a path toward the experimental realization of overlapping nanowires.

In this paper, we analytically and numerically investigate the plasmonic interaction between overlapping nanowires with and without structure singularities. Note that there has been in the past some analytical work about interacting nanowires, ${ }^{16-18}$ but the authors failed somehow to provide a physical interpretation of their analytical calculations and did not point out the contribution of surface plasmons. In this article, the conformal transformation strategy is used to derive the optical response of

\author{
*Address correspondence to \\ a.aubry@imperial.ac.uk. \\ Received for review October 19, 2010 \\ and accepted December 10, 2010. \\ Published online December 29, 2010. \\ $10.1021 / \mathrm{nn} 102819 \mathrm{p}$ \\ () 2011 American Chemical Society
}




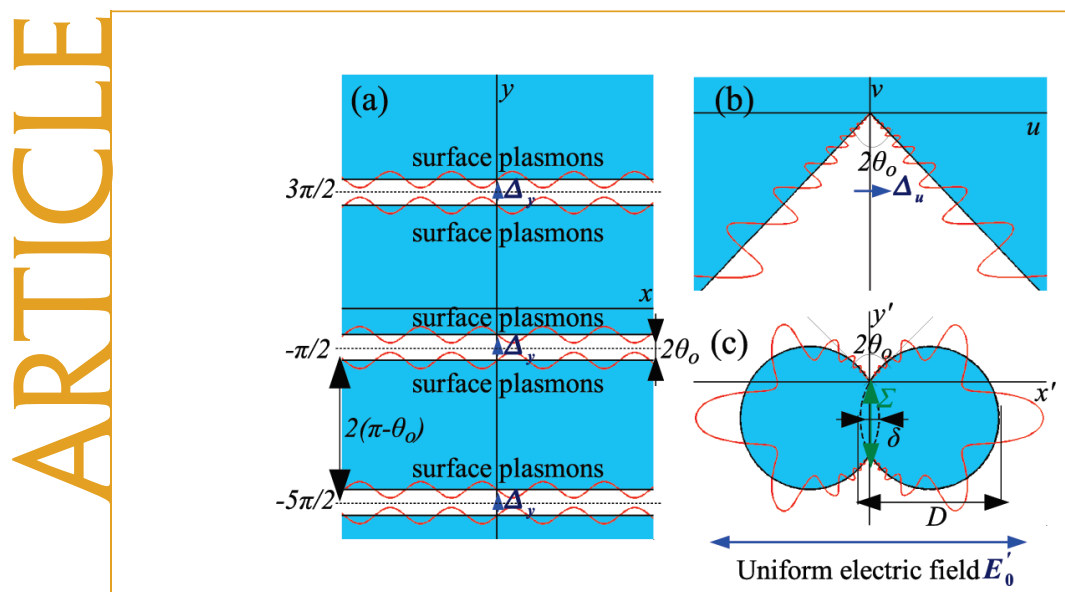

Figure 1. Periodic metallic slabs of thickness $2\left(\boldsymbol{\pi}-\boldsymbol{\theta}_{0}\right)$ separated by vacuum layers of thickness $2 \boldsymbol{\theta}_{0}$ can support surface plasmons that couple to an array of line dipoles $\Delta$. These dipoles are oriented along the $y$-direction, and the array pitch is $2 \pi$. (b) Transformed material (a) following eq 1 is a wedge-like plasmonic structure with a vertex angle $2 \theta_{0}$. The array of dipoles, $\Delta$, is transformed into a single dipole, $\Delta^{\prime}$, oriented along the $u$-axis. (c) Transformed material (b) following eq 2 is a pair of overlapping cylinders of diameter $D$, with an overlap distance $\delta$ and area of contact $\Sigma$. The dipole source $\Delta^{\prime}$ is transformed into a uniform electric field $\mathbf{E}_{{ }_{0}}$.

overlapping nanowires in the quasi-static limit. This approach provides both a qualitative and quantitative analysis of the propagation of surface plasmons in such singular structures. The system of overlapping nanowires is shown to be closely related to the case of metallic tips or wedges. ${ }^{9}$ Surface plasmon excitations are shown to exhibit a lower bound cutoff frequency, which blue-shifts with increasing overlap distance. Our approach allows one to describe theoretically the smooth transition between the ultra-broad-band response of kissing nanowires ${ }^{6,7}$ and the highly resonant feature of a single metallic nanoparticle. Below a certain critical frequency, the induced electric field exhibits a divergence at these singularities. Then, the effect of radiative losses is investigated by means of numerical simulations. Even when the structure dimension becomes comparable to the wavelength the band gap properties of the device are conserved. At last, the issue of the structure singularities is addressed. Slightly rounding off the singularities does not modify significantly the cutoff absorption behavior predicted theoretically, provided that the overlap distance is not too small. The light harvesting performance of overlapping nanowires is thus quite robust relative to potential sample imperfections. On the contrary, the divergence of the field predicted analytically disappears as soon as the singularities are blunted. Nevertheless, the field enhancement induced by a nonsingular nanostructure can still be significant.

\section{RESULTS AND DISCUSSION}

Theory. In this section, conformal mapping is adopted to solve analytically the overlapping nanowires problem. It consists in finding a conformal transformation that maps the overlapping nanowires problem onto a simple plasmonic slab geometry. The corresponding transformation is first described and allows to describe qualitatively the physics of the propagation of surface plasmons in overlapping nanowires. Then the problem is solved in the slab frame, which allows to deduce the optical response of overlapping metallic nanowires with equal size in the quasi-static limit. The light harvesting and nanofocusing properties of this device are discussed in detail.

Conformal Transformation. Our canonical system is an array of line dipoles aligned along the $y$-axis placed between periodic metallic films of thickness $2\left(\pi-\theta_{0}\right)$ (Figure 1a). This system is repeated along the $y$-direction with a period $2 \pi$. Now apply the following conformal transformation

$$
w=r_{\mathrm{o}} \exp (z)
$$

where $z=x+i y$ and $w=u+i v$ are the usual complex number notations. The transformed material consists of a wedge structure whose vertex is at the origin. The vertex angle is of $2 \theta_{0}$ (Figure $1 \mathrm{~b}$ ). With regard to the transformation of the source, the array of dipoles is transformed into a single dipole $\Delta^{\prime}=r_{0} \Delta$ aligned along the $u$-axis and placed at the point $w_{0}=-i r_{0}$. Note that this transformation has been already widely studied in ref 9. The pair of overlapping cylinders can be derived by applying the following conformal transformation to the wedge-like structure (see Figure 1c)

$$
z^{\prime}=\frac{g^{2}}{w+i r_{0}}=\frac{g^{2}}{r_{0}(\exp (z)+i)}
$$

where $z^{\prime}=x^{\prime}+i y^{\prime}$ is the usual complex number notation. The diameter of the two cylinders is given by

$$
D=\frac{g^{2}}{r_{0} \sin \theta_{0}}
$$

The overlap distance $\delta$ between the two cylinders can be expressed as

$$
\delta=D\left(1-\cos \theta_{0}\right)
$$

The contact area $\Sigma$ between the two cylinders is given by

$$
\Sigma=D \sin \theta_{0}=\frac{g^{2}}{r_{\mathrm{o}}}
$$

We also define a key parameter

$$
\rho=\frac{\Sigma}{D}=\sin \theta_{0}
$$

which is the ratio between the contact area $\Sigma$ and the diameter of the cylinders, $D$.

The dipole $\Delta^{\prime}=r_{0} \Delta$ is transformed into a uniform electric field in the transformed geometry, ${ }^{6-9}$

$$
\mathbf{E}_{0}^{\prime}=\frac{\Delta}{2 \pi \varepsilon_{0}} \frac{r_{0}}{g^{2}} \mathbf{e}_{\mathbf{x}^{\prime}}
$$


with $\mathbf{e}_{\mathbf{x}^{\prime}}$ the unitary vector along the $x^{\prime}$-axis. Under the quasi-static approximation, this uniform electric field can be taken as due to an incident plane wave in the near-field of the nanostructure. We make the choice of an incident electric field $\mathbf{E}^{\prime}{ }_{0}$ polarized along $x^{\prime}$. Actually, this polarization is by far more efficient to excite surface plasmon modes than a transverse polarization (along $\left.y^{\prime}\right) .6,7$ Note that, in the literature, most of the experimental works dealing with metallic nanowires consider surface plasmons propagating along the nanowire axis, ${ }^{15}$ contrary to the configuration studied here. We shall assume that the dimensions of the cylinders pair is sufficiently small such that surface plasmon modes are well described in the near-field approximation. In this case, the dielectric properties of the nanostructure are the same as those of the slab from which it is derived. Also preserved under the transformation is the electrostatic potential.

The mathematics of the conformal transformation closely links the physics at work in each of the very different geometries (Figure 1). Solving the relatively tractable slab problem solves the overlapping nanowires problem. It also provides a physical picture for the propagation of surface plasmons in overlapping nanowires. As shown by Figure 1c, surface plasmons are excited on the two diametrically opposite sides of the overlapping nanowires. As they propagate along the nanowires, their wavelength and velocity decrease until vanishing at the structure singularities. This leads to a drastic field enhancement in the vicinity of these singularities and even to its divergence if dissipation losses are moderate, as we will see in the following. After this brief qualitative account, we now present the details of our analytical calculations, solving first the problem in the slab geometry.

Coupling of Each Dipole to Surface Plasmons Supported by Periodic Metallic Films. The coupling of each dipole to the stack of metallic films is first addressed (Figure 1a), assuming that the Laplace's equation is obeyed. This problem has already been addressed in a more general case by Luo et al. ${ }^{9}$ Here, the symmetry of the system makes the problem simpler and allows one to derive more concise and accessible analytical results.

The position of the $n$th dipole along the $y$-axis is $y_{n}$ $=(2 n+1) \pi / 2$. As the system is $2 \pi$-periodic along the $y$-direction, we can solve the problem for $-\theta_{0}<y-y_{n}$ $<2 \pi-\theta_{0}$, and we will then deduce the solution in all of the space. Each dipole $\Delta$ consists of two line charges. We wish to calculate the potential $\phi$ induced by the metallic sheets surrounding this dipole. This can be done by expanding the incident field $\phi_{0}$ of the $n$th dipole as a Fourier series in $x$ :

$$
\begin{aligned}
\phi_{0}(x, y)=- & \frac{1}{2 \pi \varepsilon_{0}} \frac{\Delta\left(y-y_{n}\right)}{x^{2}+\left(y-y_{n}\right)^{2}}= \\
& \frac{1}{2 \pi} \int \mathrm{d} k \phi_{0}(k) e^{i k x}, \text { for }\left|y-y_{n}\right|<\theta_{0}
\end{aligned}
$$

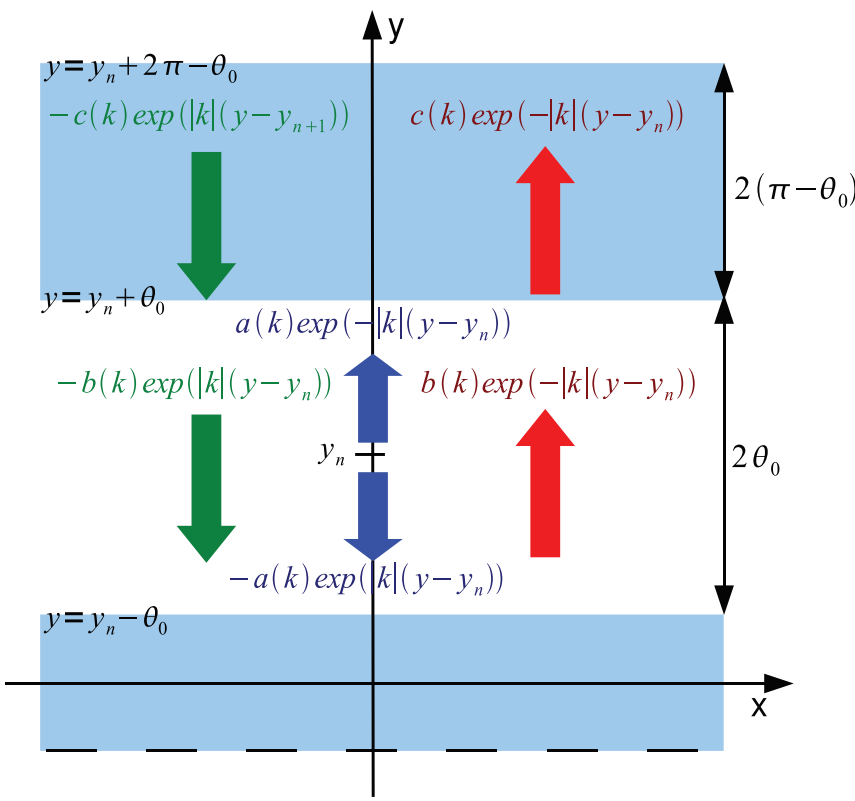

Figure 2. Sketch of the partial waves present in the structure around the $n$th dipole $\Delta$ located at $y=y_{n}$.

$\phi_{0}(k)$ can be found by making a Fourier transform at an arbitrary position $y$ :

$$
\begin{aligned}
\phi_{\mathrm{o}}(k)= & \int \phi_{\mathrm{o}}(x, y) e^{-i k y} \mathrm{~d} y= \\
& a(k) \operatorname{sgn}\left[y-y_{n}\right] e^{-|k|\left|y-y_{n}\right|}, \text { with } a(k)=-\frac{\Delta}{2 \varepsilon_{0}}
\end{aligned}
$$

The next step of our calculation consists of deriving the field $\phi(k)$ induced by the metal slabs surrounding the dipole. Due to the odd parity of the incident potential $\phi_{o}(k)$ relative to the dipole position $y_{n}$ and the symmetry of the system, the induced field $\phi(k)$ is also of odd parity relative to the dipole position. As illustrated by Figure 2, this field can be expressed as follows:

$$
\begin{aligned}
& \phi(k)= \\
& \begin{cases}b(k) e^{-|k|\left(y-y_{n}\right)}-b(k) e^{|k|\left(y-y_{n}\right)}, & \text { for }\left|y-y_{n}\right|<\theta_{0} \\
c(k) e^{-|k|\left(y-y_{n}\right)}-c(k) e^{|k|\left(y-y_{n+1}\right)}, & \text { for } \theta_{0}<y-y_{n}<2 \pi-\theta_{0}\end{cases}
\end{aligned}
$$

The two unknowns $b(k)$ and $c(k)$ are then determined by the boundary conditions at the metallic slab interface $\left(y=y_{n}+\theta_{0}\right)$. They derive from the conservation of the parallel component of the electric field and of the normal component of the displacement field

$$
\begin{aligned}
& a(k) e^{-|k| \theta_{0}}+b(k)\left(e^{-|k| \theta_{0}}-e^{|k| \theta_{0}}\right)=c(k)\left(e^{-|k| \theta_{0}}-e^{-|k|\left(2 \pi-\theta_{0}\right)}\right) \\
& a(k) e^{-|k| \theta_{0}}+b(k)\left(e^{-|k| \theta_{0}}+e^{|k| \theta_{0}}\right)=\varepsilon c(k)\left(e^{-|k| \theta_{0}}+e^{-|k|\left(2 \pi-\theta_{0}\right)}\right)
\end{aligned}
$$

Solving these two equations provides the following results, using the expression of $a(k)$ (eq 9)

$$
\begin{aligned}
& b(k)= \\
& -\frac{\Delta}{4 \varepsilon_{0}} \frac{\exp \left(-|k| \theta_{0}\right)\left[\varepsilon-\tanh \left(|k|\left(\pi-\theta_{0}\right)\right)\right]}{\cosh \left(|k| \theta_{0}\right)\left[\tanh \left(|k|\left(\pi-\theta_{0}\right)\right)+\varepsilon \tanh \left(|k| \theta_{0}\right)\right]} \\
& \text { VOL. } 5 \cdot \text { NO. } 1 \cdot 597-607 \cdot 2011
\end{aligned}
$$




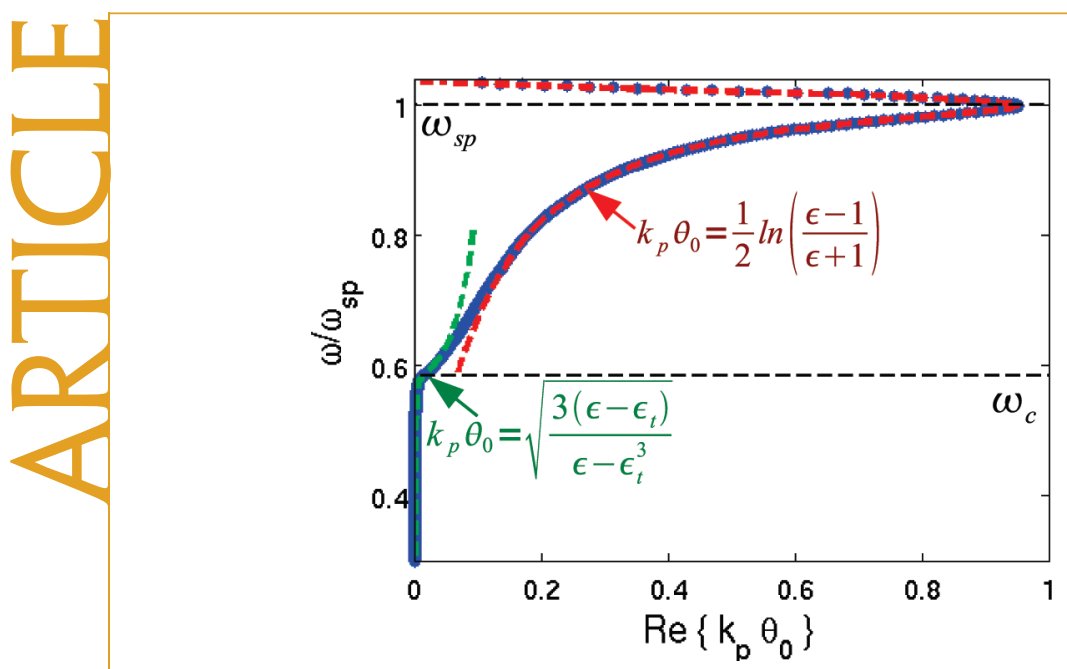

Figure 3. Dispersion curve.

$c(k)=$

$$
-\frac{\Delta}{4 \varepsilon_{0}} \frac{\exp (|k| \pi)}{\cosh \left(|k| \theta_{0}\right) \cosh \left(|k|\left(\pi-\theta_{0}\right)\right)\left[\tanh \left(|k|\left(\pi-\theta_{0}\right)\right)+\varepsilon \tanh \left(|k| \theta_{0}\right)\right]}
$$

The dispersion of the excitations can be found from the condition that $b(k)$ and $c(k)$ diverges

$$
\tanh \left(|k|\left(\pi-\theta_{0}\right)\right)=-\varepsilon \tanh \left(|k| \theta_{0}\right)
$$

This equation cannot be solved analytically. Nevertheless, two asymptotic solutions can be derived

$$
\begin{aligned}
& k_{p} \theta_{0}=\sqrt{\frac{3\left(\varepsilon-\varepsilon_{c}\right)}{\varepsilon-\varepsilon_{c}^{3}}}, \text { for }\left|k_{\mathrm{p}}\right| \theta_{0} \ll 1, \\
& \text { i.e., when } \operatorname{Re}\{\varepsilon\} \rightarrow \varepsilon_{\mathrm{c}} \\
& k_{\mathrm{p}} \theta_{0}=\frac{1}{2} \ln \left(\frac{\varepsilon-1}{\varepsilon+1}\right), \text { for }\left|k_{\mathrm{p}}\right|\left(\pi-\theta_{0}\right) \gg 1, \\
& \text { i.e., when }-\varepsilon_{\mathrm{c}}\left|\ln \left(\frac{\varepsilon-1}{\varepsilon+1}\right)\right| \gg 1
\end{aligned}
$$

where we have introduced, $\varepsilon_{c}$, a threshold value for

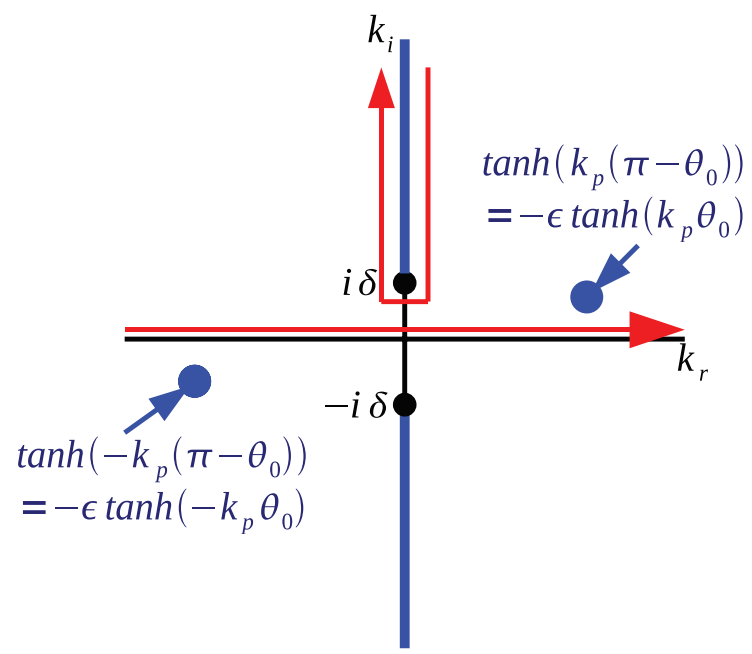

Figure 4. Analytic structure of the integrand of eq 17. There are two cuts running from $-i \delta$ and $+i \delta$ (blue line). There are also two poles if $\varepsilon<0$ (blue disks). If $\varepsilon>0$, these poles vanish in the cuts and give no contribution.

the permittivity

$$
\varepsilon_{c}=\frac{\theta_{0}-\pi}{\theta_{0}}
$$

This threshold value is of particular importance for a metal of permittivity $\varepsilon<\varepsilon_{\mathrm{c}}$, and no surface plasmon modes can be supported by the stack of metal slabs. ${ }^{9}$ This is confirmed by Figure 3 which shows the dispersion relation of SPPs derived from eq 13. For this figure as well as in the following of this section, the metal is assumed to be silver with a surface plasmon frequency $\omega_{\mathrm{sp}}=3.67 \mathrm{eV}$ and permittivity taken from Johnson and Christy. ${ }^{19}$ The dispersion curve displays two branches of surface plasmon excitations, namely, the even branch for $\omega<\omega_{\text {sp }}$ and the odd branch which spans the frequency range $\omega_{\text {sp }}<\omega<\omega_{\mathrm{p}}$ (with $\omega_{\mathrm{p}}$ as the bulk plasmon frequency). However, contrary to a finite metal-instulator-metal structure, the periodicity of the system induces here a lower bound cutoff frequency, $\omega_{c}$, which arises when $\operatorname{Re}\{\varepsilon\}=\varepsilon_{c}$. Figure 3 shows that the two asymptotic solutions of the dispersion relation (eqs 14 and 15) provides an analytical solution over a major part of the spectrum.

Now that the condition of divergence for the coefficients $b(k)$ and $c(k)$ have been derived, the potential can be solved in the real space. An inverse Fourier transform of the induced potential derived in the k-space leads to the solution in the real space

$\phi(x, y)=-\frac{1}{2 \pi} \times$

$\begin{cases}\int 2 b(k) \sinh \left(|k|\left(y-y_{n}\right)\right) e^{i k x} \mathrm{~d} k, & \text { for }\left|y-y_{n}\right|<\theta_{0} \\ \int 2 c(k) e^{-|k| \pi} \sinh \left(|k|\left(y-y_{n}-\pi\right)\right) e^{i k x} \mathrm{~d} k, & \text { for } \theta_{0}<y-y_{n}<2 \pi-\theta_{0}\end{cases}$

To perform this integration, we write

$$
|k|=\lim _{\delta \rightarrow \infty}\left(k^{2}+\delta^{2}\right)^{1 / 2}
$$

The analytic structure of eq 17 is shown in Figure 4. There are two branch cuts running from $-i \delta$ and $+i \delta$, ensuring the analytical continuity of the integrand. The integral is dominated by the poles close to the real axis which correspond to surface plasmon modes carrying away energy to infinity. The cuts correspond to lossy or creeping surface waves which, if $\varepsilon$ is real, dissipate no energy..$^{10}$ Therefore, in the limit of real $\varepsilon$, our expression for dissipation will be exact, but otherwise only approximate. From now on, we will only consider the frequency band below the surface plasmon frequency, $\omega<\omega_{\text {sp, }}$ for which $\varepsilon<-1$. Actually, beyond $\omega_{\text {sp }}$ the imaginary part $\varepsilon_{l}$ of the metal permittivity becomes comparable to its real part $\varepsilon_{R}$, and the contribution from the cuts shown in Figure 4 is no longer negligible.

The calculation of the integral in eq 17 leads to 


$$
\phi\left(\left|y-y_{n}\right|<\theta_{0}\right)=i \frac{\Delta}{2 \varepsilon_{0} \theta_{0}} \Gamma \sinh \left[k_{\mathrm{p}}\left(y-y_{n}\right)\right] e^{i k_{p}|x|}
$$

$$
\text { with } \Gamma=\frac{\varepsilon \cosh ^{-1}\left(k_{\mathrm{p}} \theta_{0}\right) \exp \left(-k_{\mathrm{p}} \theta_{0}\right)\left[1+\tanh \left(k_{\mathrm{p}} \theta_{0}\right)\right]}{\varepsilon-\varepsilon_{\mathrm{c}}+\varepsilon\left(\varepsilon \varepsilon_{\mathrm{c}}-1\right) \tanh ^{2}\left(k_{\mathrm{p}} \theta_{0}\right)}
$$$$
\phi\left(\theta_{0}<y-y_{n}<2 \pi-\theta_{0}\right)=
$$

$$
\begin{array}{r}
i \frac{\Delta}{2 \varepsilon_{0} \theta_{0}} \Gamma^{\prime} \sinh \left[k_{\mathrm{p}}\left(y-y_{n}-\pi\right)\right] e^{i k_{\mathrm{p}}|x|} \\
\text { with } \Gamma^{\prime}=\frac{\cosh ^{-1}\left(k_{\mathrm{p}} \theta_{0}\right) \cosh ^{-1}\left(\varepsilon_{\mathrm{c}} k_{\mathrm{p}} \theta_{0}\right)}{\varepsilon-\varepsilon_{\mathrm{c}}+\varepsilon\left(\varepsilon \varepsilon_{\mathrm{c}}-1\right) \tanh ^{2}\left(k_{\mathrm{p}} \theta_{0}\right)}
\end{array}
$$

The expression of $\Gamma$ that will be useful in the following can be simplified considering the asymptotic limits of $k_{\mathrm{p}}$ (eqs 14 and 15)

$$
\begin{gathered}
\Gamma \sim-2 \frac{\varepsilon}{\varepsilon-\varepsilon_{\mathrm{c}}} \text { when } \operatorname{Re}\{\varepsilon\} \rightarrow \varepsilon_{\mathrm{c}} \\
\Gamma \sim 1 \text { when }-\varepsilon_{\mathrm{c}}\left|\ln \left(\frac{\varepsilon-1}{\varepsilon+1}\right)\right| \gg 1
\end{gathered}
$$

From the expression of the induced potential $\phi$ for $\mid y$ $-y_{n} \mid<\theta_{0}$ (eq 18), we can deduce the electric field induced by the metal slabs on each dipole

$$
\mathbf{E}\left(z=i y_{n}\right)=-\nabla \phi=-i \frac{\Delta}{2 \varepsilon_{0} \theta_{0}} \Gamma k_{\mathrm{p}}
$$

The electric field induced at the dipoles is of particular interest, since it directly provides the energy dissipated in the surface plasmon mode.

Absorption Cross Section. As shown by previous studies, 7,8 energy dissipation is the same in the slab and transformed geometries. The dipole energy pumped into surface plasmons in the metal slabs (Figure 1a) maps directly onto the power absorbed by the overlapping cylinder pair from the incident electric field $\mathbf{E}^{\prime}{ }_{0}$ in the transformed frame (Figure 1c):

$$
P_{\mathrm{a}}=-\frac{\omega}{2} \operatorname{lm}\left\{\Delta^{*} \mathbf{e}_{\mathbf{x}} \cdot \mathbf{E}\left(z=i y_{n}\right)\right\}
$$

If we inject the expression of $\mathbf{E}(z=0)$ (eq 24) into the last equation, replace $\Delta$ and $g^{2} / r_{0}$ with $\mathbf{E}^{\prime}{ }_{0}$ (eq 7) and $\Sigma$ (eq 5) and renormalize it by the incoming flux $P_{\text {in }}=$ $\varepsilon_{0} c_{0}\left|\mathbf{E}^{\prime}\right|^{2} / 2$, we can derive the absorption cross section $\sigma_{\mathrm{a}}=P_{\mathrm{a}} / P_{\mathrm{in}}$ of the overlapping cylinders pair

$$
\sigma_{\mathrm{a}}=2 \pi^{2} k_{0} \frac{\Sigma^{2}}{\theta} \operatorname{Re}\left\{k_{\mathrm{p}} \Gamma\right\}
$$

where $k_{0}=\omega / c_{0}$ is the wavenumber in vacuum. This expression of $\sigma_{\mathrm{a}}$ can be made more explicit by considering the asymptotic limits of $k_{\mathrm{p}}$ (eqs 14 and 15) and $\Gamma$ (eqs 22 and 23) and by replacing $\Sigma$ and $\theta$ by $D$ and $\rho$ (eq 6)
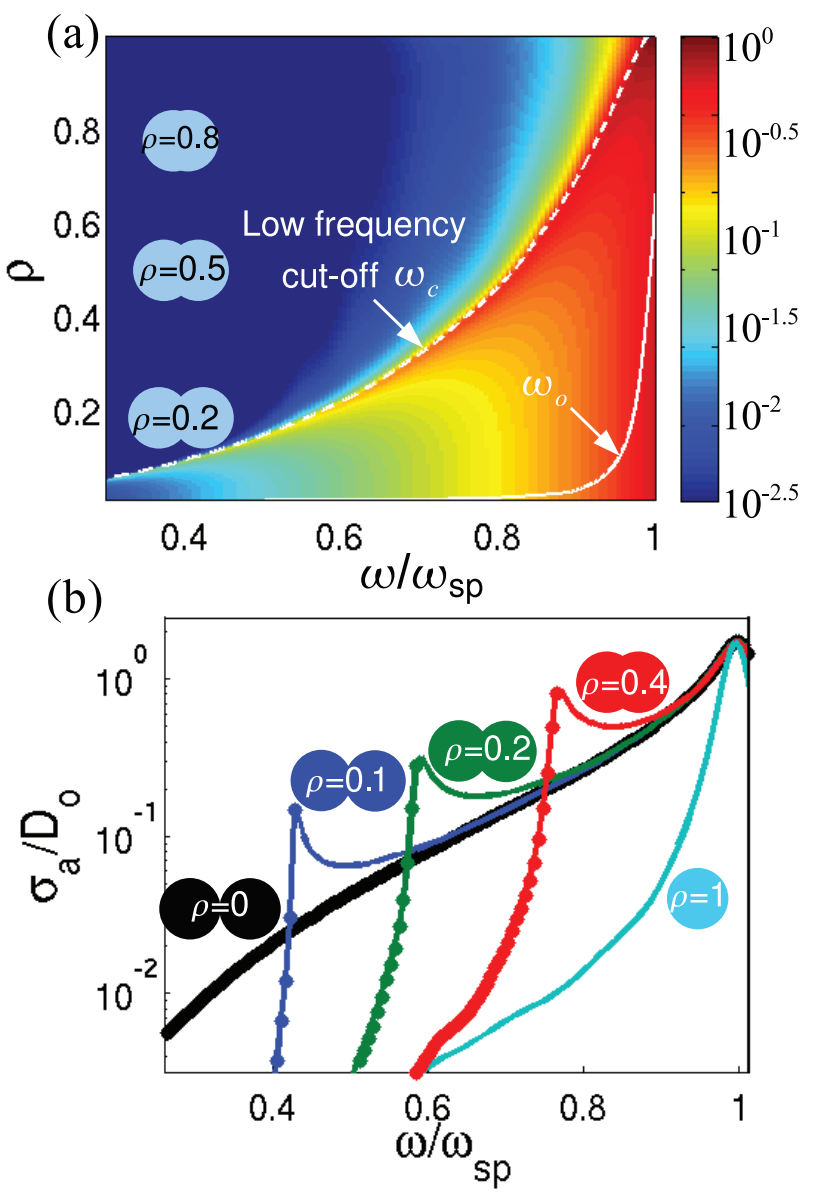

Figure 5. (a) Absorption cross section $\sigma_{\mathrm{a}}$ (eq 26) normalized by the physical cross section $D_{0}$ as a function of $\rho$ and frequency for an overlapping cylinders' pair of size $D_{0}=20 \mathrm{~nm}$. The white dashed line represents the low-frequency cutoff $\omega_{c}$. The white continuous line represents the frequency $\omega_{0}$ (eq 34). The color bar is in log scale. (b) Same quantity is displayed as a function of frequency for different values of $\rho=0.1$ (blue), 0.2 (green), and 0.4 (red). The low-frequency asymptote (colored dots, eq 27) is displayed for each value of $\rho$. The highfrequency asymptote (black dots, eq 28) is also shown and corresponds to the kissing cylinders case $(\rho=0)$. At last, the single cylinder case is also shown for comparison ${ }^{20}$ (cyan continuous line). For both panels, the metal is assumed to be silver with a surface plasmon frequency $\omega_{\text {sp }}=3.67 \mathrm{eV}$ and permittivity taken from Johnson and Christy. ${ }^{19}$

$$
\begin{array}{r}
\sigma_{\mathrm{a}} \sim \pi^{2} \sqrt{3} k_{0} \frac{\rho^{2}}{\arcsin ^{2}(\rho)} D^{2} \operatorname{Re}\left\{\sqrt{\frac{\varepsilon_{\mathrm{c}}}{1-\varepsilon_{\mathrm{c}}^{2}}} \frac{1}{\sqrt{\varepsilon-\varepsilon_{\mathrm{c}}}}\right\} \\
\text { when } \operatorname{Re}\{\varepsilon\} \rightarrow \varepsilon_{\mathrm{c}}
\end{array}
$$

$$
\begin{aligned}
\sigma_{\mathrm{a}} \sim \pi^{2} k_{0} \frac{\rho^{2}}{\arcsin ^{2}(\rho)} D^{2} \operatorname{Re}\left\{\ln \left(\frac{\varepsilon-1}{\varepsilon+1}\right)\right\} \\
\text { when }-\varepsilon_{\mathrm{c}}\left|\ln \left(\frac{\varepsilon-1}{\varepsilon+1}\right)\right| \gg 1
\end{aligned}
$$

The first asymptote of $\sigma_{a}$ (eq 27) displays a square-root singularity at the cutoff frequency $\omega_{c}$, for which $\operatorname{Re}\{\varepsilon\}$ $=\varepsilon_{c}$. The second asymptote (eq 28) applies for larger frequencies than $\omega_{\mathrm{c}}$ and is strictly identical to the expression derived for kissing cylinders. ${ }^{6,7}$ Figure 5 a displays the absorption cross section of overlapping 

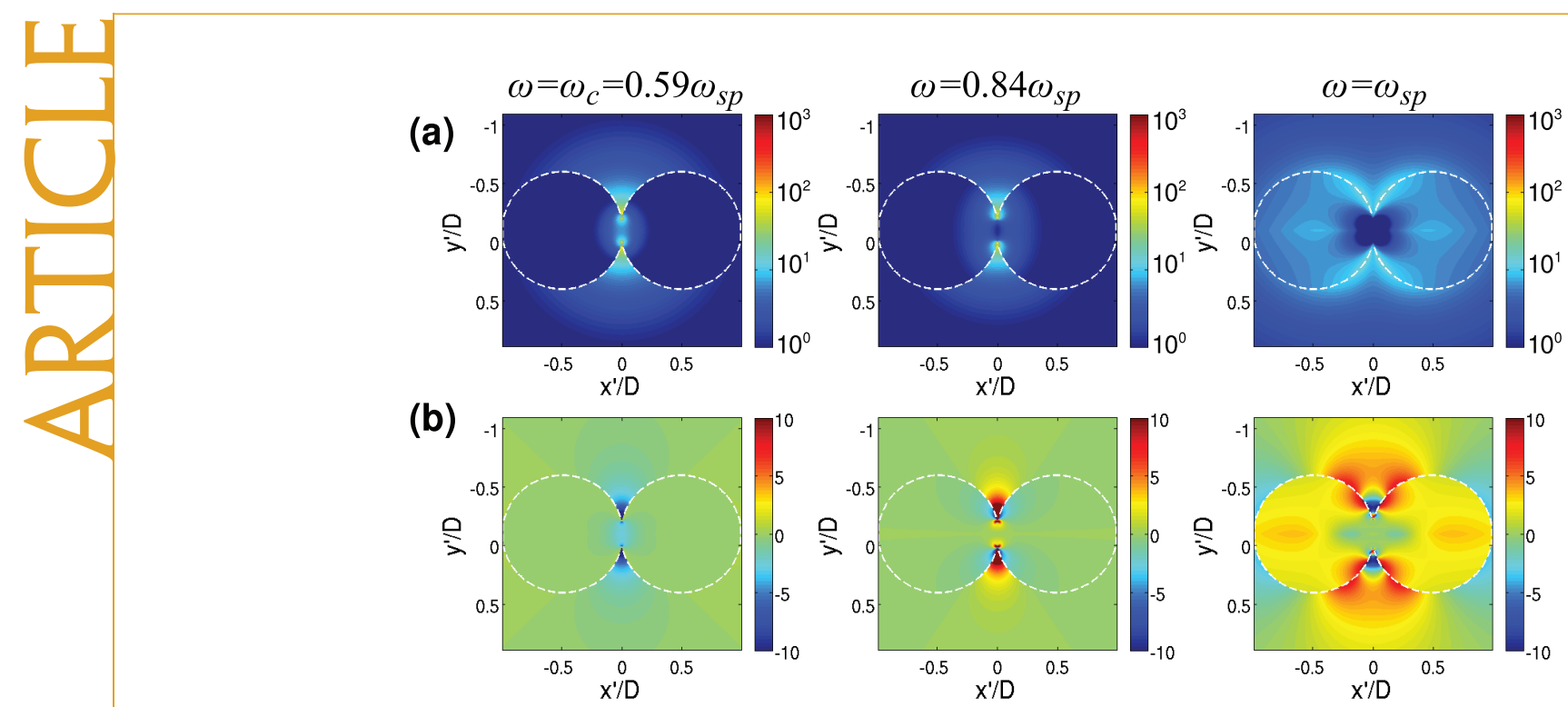

Figure 6. Distribution of the electric field in overlapping cylinders for $r=0.2$ shown at different frequencies. (a) Absolute value of the electric field $\left|E^{\prime}\right|$ normalized by the incoming field $\left|E_{0}^{\prime}\right|$ (polarized along $x^{\prime}$ ). The color scale is logarithmic and restricted $\left[1 \times 10^{3}\right.$ ], but note that the field magnitude can larger especially at the vicinity of the structure singularity where the electric field diverge for the left and middle columns. (b) Amplitude of the real part of $E^{\prime}{ }_{x^{\prime}}$ normalized by the incoming field $E_{0}^{\prime}$ (polarized along $x^{\prime}$ ). The color scale is linear and restricted to [-10 10], but note that the field magnitude can by far larger especially at the structure singularity.

nanowires normalized by the overall physical cross section $D_{0}=2 D-\delta$ as a function of frequency and the ratio $\rho$ between the contact area $\Sigma$ and the nanowire diameter $D$. Figure $5 \mathrm{~b}$ displays the frequency dependence of $\sigma_{\mathrm{a}} / D_{\mathrm{o}}$ for different values of $\rho$. The two asymptotes derived in eqs 27 and 28 are also shown on that graph.

As shown in Figure 5, the absorption spectrum is strongly dependent on the overlap distance between the two nanowires. Actually, the ratio $\rho=\Sigma / D$ is directly related to the angle of contact $\theta_{0}$ between the two nanowires (eq 6), which governs the cutoff frequency $\omega_{c}$ (eq 16). A squeezed metallic wedge $\left(\theta_{0} \rightarrow 0\right)$ can support surface plasmon modes over a broad-band spectrum $\left(\omega_{c} \rightarrow 0\right)$, whereas large angles $\theta_{0}$ imply an extremely narrow line width $\left(\omega_{\mathrm{c}} \rightarrow \omega_{\mathrm{sp}}\right)$. Hence, three distinct regimes can be distinguished:

- Kissing cylinders regime $(\rho \rightarrow 0)$ : this regime has already been widely studied previously. ${ }^{6,7}$ The absorption spectrum of kissing nanowires corresponds to the high-frequency asymptote derived in eq 28 (see Figure $5 \mathrm{~b}$ ). In that case, the cutoff frequency $\omega_{c}$ is zero and the absorption cross section displays a continuous and broad-band absorption spectrum over the whole visible and near-infrared spectra.

- Overlapping regime $(0<\rho<1)$ : when the angle of contact $\theta_{0}$ (or $\rho$ ) increases, the cutoff frequency $\omega_{c}$ blue-shifts, which limits the bandwidth of the light harvesting process. An absorption peak is observed around $\omega_{c}$ and its line shape is well-predicted by eq 27 for $\omega<\omega_{\mathrm{c}}$. When $\omega \rightarrow \omega_{\mathrm{sp}}$, the device behaves like kissing cylinders (eq 28) in terms of light harvesting (see Figure 5b). The comparison stops here since the electric field distribution is on the contrary dramatically different between kissing and overlapping cylinders, as we will see later. Anyway, for small overlap ( $\rho<0.5$ ), the absorption spectrum exhibits an interesting light harvesting feature with a flat and broad-band absorption spectrum between $\omega_{\mathrm{c}}$ and $\omega_{\text {sp }}$.

- Single nanowire regime ( $\rightarrow 1$ ): when $\rho \rightarrow 1$, the two nanowires merge into a single one. The absorption spectrum exhibits then one sharp resonance at the surface plasmon frequency $\omega_{\text {sp }}$ (see Figure 5b).

In contrast to the kissing nanowires $(\rho=0)$, overlapping nanowires show a clear-cutoff frequency $\omega_{c}$ in their absorption spectrum. This cutoff frequency can be adjusted by tailoring the overlap distance between the two nanowires. This opens nice perspectives for the design of plasmonic band gap filters.

Electric Field in the Transformed Geometry. Under the conformal transformation, the electrostatic potential is preserved. The electric field $\mathbf{E}^{\prime}\left(x^{\prime}, y^{\prime}\right)$ in the transformed geometry can then be easily deduced from the potential derived in the slab geometry

$$
\begin{aligned}
& E_{x^{\prime}}^{\prime}=-\frac{\partial \phi^{\prime}}{\partial z^{\prime}} \frac{\partial z}{\partial x^{\prime}}-\frac{\partial \phi^{\prime}}{\partial z^{\prime *}} \frac{\partial z^{\prime *}}{\partial x^{\prime}}=-\frac{\partial \phi^{\prime}}{\partial z^{\prime}}-\frac{\partial \phi^{\prime}}{\partial z^{\prime *}} \\
& E_{y^{\prime}}^{\prime}=-\frac{\partial \phi^{\prime}}{\partial z^{\prime}} \frac{\partial z}{\partial y^{\prime}}-\frac{\partial \phi^{\prime}}{\partial z^{\prime *}} \frac{\partial z^{\prime *}}{\partial y^{\prime}}=-i \frac{\partial \phi^{\prime}}{\partial z^{\prime}}+i \frac{\partial \phi^{\prime}}{\partial z^{\prime *}}
\end{aligned}
$$

Using the expression of the potential $\phi$ given in eqs $18-20$, the electric field $\mathbf{E}^{\prime}$ can be expressed as a function of $\mathbf{E}^{\prime}{ }_{0}$ (eq 7), $\Sigma$ (eq 5), and $\theta_{0}$ :

- For $\left|z^{\prime}+(D-\delta+i \Sigma) / 2\right|>D / 2$ or $\mid z^{\prime}-(D-\delta-$ $i \Sigma) / 2 \mid>D / 2$ (i.e., outside the cylinders): 


$$
\begin{gathered}
E_{x^{\prime}}^{\prime}=i E_{0} \frac{\pi}{2} \frac{\Sigma^{2}}{\theta_{0}} k_{\mathrm{p}} \Gamma\left[\frac{1}{z^{\prime * 2}}\left(1-i \frac{\Sigma}{z^{\prime *}}\right)^{ \pm i k_{\mathrm{p}}-1}+\right. \\
\left.\frac{1}{\mathrm{z}^{\prime 2}}\left(1+i \frac{\Sigma}{z^{\prime}}\right)^{ \pm i k_{\mathrm{p}}-1}\right] \\
E_{y^{\prime}}^{\prime}=E_{0} \frac{\pi}{2} \frac{\Sigma^{2}}{\theta_{0}} k_{\mathrm{p}} \Gamma\left[\frac{1}{z^{\prime * 2}}\left(1-i \frac{\Sigma}{\mathrm{z}^{\prime *}}\right)^{ \pm i k_{\mathrm{p}}-1}-\right. \\
\left.\frac{1}{\mathrm{z}^{\prime 2}}\left(1+i \frac{\Sigma}{z^{\prime}}\right)^{ \pm i k_{\mathrm{p}}-1}\right]
\end{gathered}
$$

- For $\left|z^{\prime}+(D-\delta+i \Sigma) / 2\right|<D / 2$ or $\mid z^{\prime}-(D-\delta-$ $i \Sigma) / 2 \mid<D / 2$ (i.e., inside the cylinders):

$$
\begin{gathered}
E_{\chi^{\prime}}^{\prime}=-i E_{0} \frac{\pi}{2} \frac{\Sigma^{2}}{\theta_{0}} k_{\mathrm{p}} \Gamma^{\prime}\left[\frac{1}{z^{\prime * 2}}\left(-1+i \frac{\Sigma}{\mathrm{z}^{\prime *}}\right)^{ \pm i k_{\mathrm{p}}-1}+\right. \\
\left.\frac{1}{\mathrm{z}^{\prime 2}}\left(-1-i \frac{\Sigma}{z^{\prime}}\right)^{ \pm k_{\mathrm{p}}-1}\right]
\end{gathered}
$$

$$
\begin{gathered}
E_{y^{\prime}}^{\prime}=-E_{0} \frac{\pi}{2} \frac{\Sigma^{2}}{\theta_{0}} k_{\mathrm{p}} \Gamma^{\prime}\left[\frac{1}{z^{* 2}}\left(-1+i \frac{\Sigma}{z^{\prime *}}\right)^{ \pm k_{\mathrm{p}}-1}-\right. \\
\left.\frac{1}{\mathrm{z}^{\prime 2}}\left(-1-i \frac{\Sigma}{z^{\prime}}\right)^{ \pm i k_{\mathrm{p}}-1}\right]
\end{gathered}
$$

where \pm corresponds to the sign of $y^{\prime}+i \Sigma / 2$. Note that in the near-field approximation, which holds when the dimensions of the structure are less than the wavelength, the enhancement of electric field is independent of the size of the system. Figure 6 shows the result of our analytical calculation of the electric field for overlapping cylinders with $\rho=0.2$ at different frequencies.

The first column of Figure 6 corresponds to the field obtained at $\omega=\omega_{c}$. In that case, the field is extremely confined and intense in the vicinity of the structure singularities $\left(z^{\prime}=0\right.$ and $\left.z^{\prime}=-i \Sigma\right)$, that is, the points where the two nanowires merge. Actually, the field diverges at these locations. ${ }^{9}$ This can be shown by taking a careful look at the expression of the electric field (eqs 29-32). Let us consider as an example the expression of $E_{x^{\prime}}^{\prime}$ outside the nanowires (eq 29). The terms within the brackets are the products of two different powers of $z^{\prime}$. The first one scales as $z^{\prime-2}$ and accounts for the geometrical compression of surface plasmons when they approach the structure singularity. It may lead to the divergence of the electric field at this location. The second one, scaling as $\left(1+i\left(\sum / z^{\prime}\right)\right)^{ \pm i k_{\mathrm{p}}-1}$, describes the propagation and attenuation of surface plasmons when they propagate along the nanowires' surface. At the structure singularities, the product of these two terms will diverge if and only if

$$
\operatorname{Im}\left\{k_{\mathrm{p}}\right\}<1
$$

Considering the high-frequency limit of $k_{p}$ (eq 15), the field divergence condition can be rewritten as

$$
\arctan \left[\frac{2 \operatorname{lm}\{\varepsilon\}}{|\varepsilon|^{2}+1}\right]<2 \theta_{0}
$$

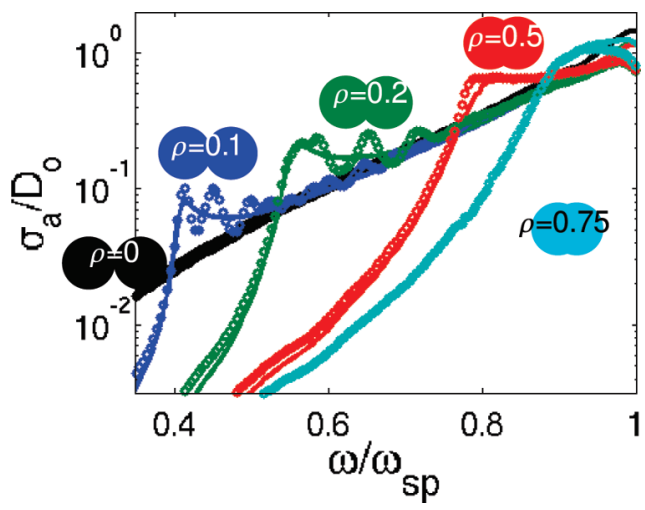

Figure 7. Comparison between theoretical (solid lines) and simulated (dots) absorption cross sections normalized by the physical cross section $D_{0}$ as a function of frequency for an overlapping nanowires' pair of size $D_{0}=20 \mathrm{~nm}$ with different values of $\rho=0$ (black), 0.1 (blue), 0.2 (green), 0.5 (red), and 0.75 (cyan). The metal is assumed to be silver with permittivity taken from Palik. ${ }^{22}$

The term on the left is directly related to the dissipation losses in the metal with the imaginary part of the permittivity. The term on the right is the vertex angle which accounts for the field compression at the structure singularities. Let us introduce $\omega_{0}$ the frequency for which the two terms of the last equation are equal. Below $\omega_{0}$, eq 34 is checked: the compression factor dominates over dissipation losses and the field diverges at the structure singularities. Beyond $\omega_{0}$, eq 34 is no longer verified: the dissipation losses are large enough to make the field vanish at the structure singularities. $\omega_{\mathrm{o}}$ and $\omega_{c}$ are shown as a function of $\rho$ and are superimposed to the absorption spectrum in Figure 5a. The comparison between the two curves shows that the frequency $\omega_{0}$ is clearly larger than the cutoff frequency $\omega_{c}$ and that both frequencies blue-shift with the overlap distance. The divergence of the electric field occurs over most of the overlapping nanowires' bandwidth.

The second column of Figure 6 confirms this divergent feature by showing the field at a frequency $\omega=$ $0.84 \omega_{\mathrm{sp}}$ for which $\omega_{c}<\omega<\omega_{0}$. Note that, at such frequency, the absorption spectrum follows the kissing cylinders asymptote (see Figure 5b). However, the electric field distribution in the near-field of the nanowires is completely different compared to a kissing cylinders configuration. In the latter case, the electric field always vanish at the structure singularity. ${ }^{6,7}$ For overlapping nanowires, the field exhibits a divergent feature similar to what happens in metallic tips or wedges. ${ }^{9}$ At last, the third column of Figure 6 shows the electric field at $\omega$ $=\omega_{\mathrm{sp}}$. In that case, dissipation losses are important and make the electric field decrease when surface plasmons approach the structure singularity. The electric field then spreads spatially over the whole nanowire surface.

This divergent feature at the singularities of the nanostructure is interesting for theoreticians but does not really make sense experimentally. First, the nonlo- 

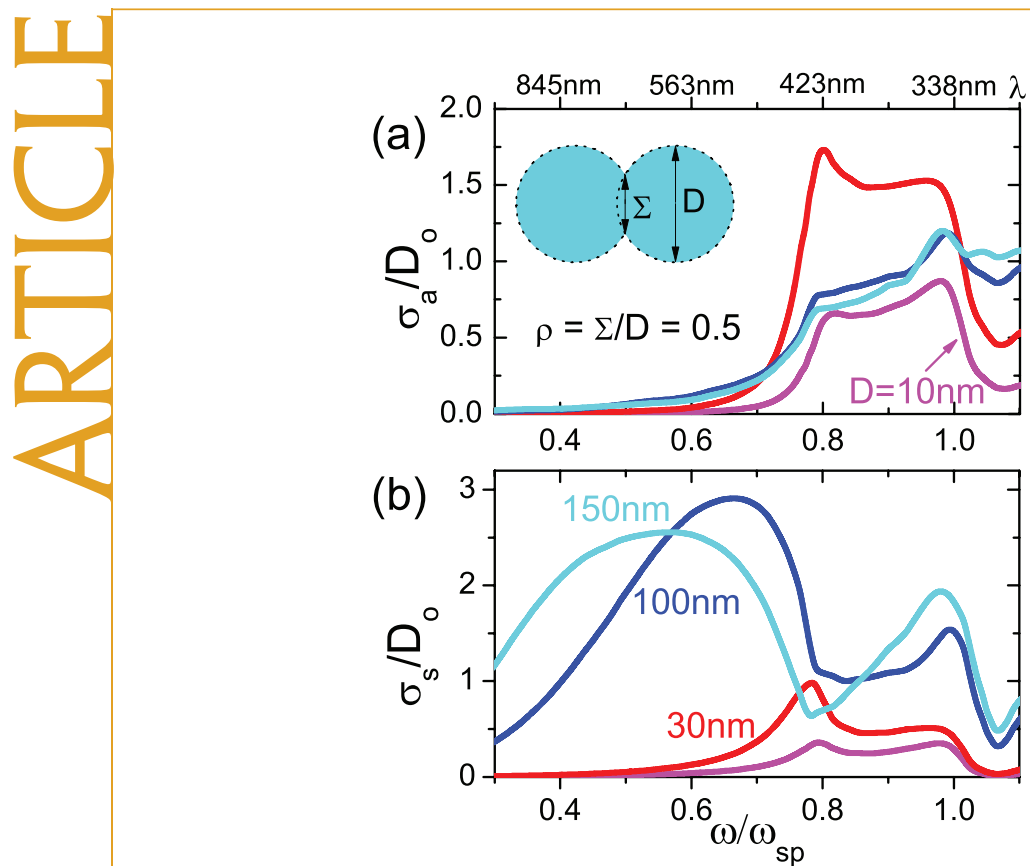

Figure 8. Simulated absorption cross section $\sigma_{\mathrm{a}}(\mathrm{a})$ and scattering cross section $\sigma_{s}(b)$ normalized by the physical cross section $D_{\mathrm{o}}$ as a function of frequency for overlapping nanowires' pairs with a constant $\rho=0.5$ but different diameters, $D=10$ (purple), 30 (red), 100 (blue), and $150 \mathrm{~nm}$ (cyan). For both panels, the metal is assumed to be silver with permittivity taken from Palik. ${ }^{22}$

cal properties of the permittivity at small length scales will prevent the electric field from increasing to infinity. ${ }^{21}$ Second, this divergent feature highly relies on the singularities of the nanostructure which will never be perfectly reproduced in experiments. Hence, a numerical study is also needed to check the validity of our analytical approach and its range of application.

Numerical Simulations. This section deals with a numerical study of the overlapping nanowires system and its confrontation with our analytic theory. The numerical method is described in details in the Methods section. The interest of the conformal transformation approach in dealing with singular structures is first highlighted by showing that numerical simulations cannot reach the same degree of precision as our analytic results. The effect of radiative losses is then investigated when the structure dimension becomes comparable to the wavelength. The influence of the structure singularities is also studied. Interestingly, the numerical simulations show that slightly rounding off the structure singularities from overlapping nanowires does not change significantly the cutoff absorption behavior predicted by theory, provided that the overlap distance is not too small ( $\rho \geq 0.5$ ). Not surprisingly, the divergent feature of the field enhancement is suppressed when structure singularities are blunted. However, numerical simulations show that it can still be drastic even in presence of sample imperfections.

Quasi-static Limit. Figure 7 compares the analytical and numerical absorption cross sections obtained for an

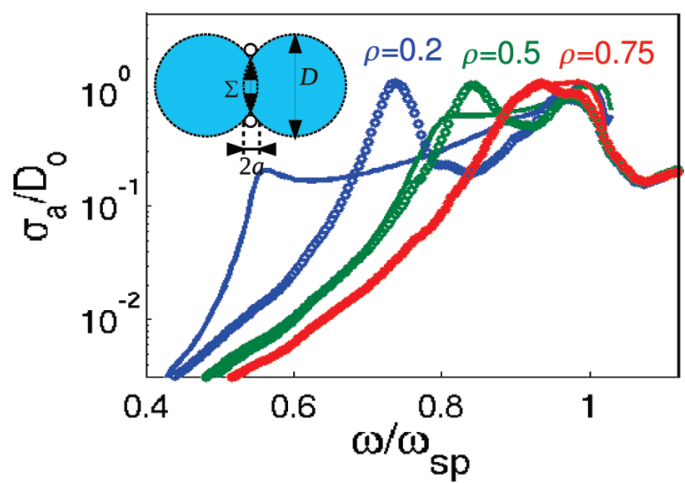

Figure 9. Simulated absorption cross section $\sigma_{\mathrm{a}}$ normalized by the physical cross section $D_{0}$ as a function of frequency for an overlapping nanowires' pair with blunted singularities compared to theoretical predictions that have involved singularities in the calculations. The two singularities of the nanowires' pair have been removed by inserting two circles of $0.2 \mathrm{~nm}$ radius as shown in the inset. The area enclosed between these circles and the nanoparticles are then filled with metal. The structure has a total dimension of $D_{0}=20$ $\mathrm{nm}$ with different values of $\rho=0.2$ (blue), 0.5 (green), and 0.75 (red).

overlapping nanowires' pair of size $D_{0}=20 \mathrm{~nm}$ with different values of $\rho$. For such dimension, the near-field approximation is valid. ${ }^{10}$ Note that for this figure as well as in the following of the study, the silver permittivity is now taken from Palik data ${ }^{22}$ and no longer from Johnson and Christy ${ }^{19}$ as in Theory. Actually, we have been unable to make the simulations converge with Johnson and Cristy data because of the strong divergence of the electric field at the structure singularities. On the contrary, the silver dielectric function from the Palik data 22 has a larger imaginary part in the frequency range of interest, which implies more dissipation losses and a less dramatic singular feature. Nevertheless, slight oscillations are still visible in the simulated absorption spectra for small values of $\rho$ compared to theory (see Figure 7). These numerical difficulties highlight the importance of developing analytical approaches to deal with such singular structures.

Radiative Losses: Negligible Absorption and Strong Scattering below the Cutoff Frequency. The radiative losses, which are not taken into account in the conformal transformation approach, are now investigated by means of numerical simulations. As shown in a previous study ${ }_{1}^{10}$ these radiative losses mainly originate from the lossy surface waves, whose contribution cannot be derived analytically in the case of overlapping nanowires. The absorption and scattering cross sections of overlapping nanowires have been computed numerically for different structure dimensions at $\rho=0.5$ (see Figure 8). The results show that radiation damping becomes important for $D \geq 30 \mathrm{~nm}$. The absorption cross section falls compared to the quasi-static prediction: the theory predicts a scaling of $\sigma_{\mathrm{a}}$ as $D^{2}$ (eq 27), which is clearly not the case for $D \geq 30 \mathrm{~nm}$ (see Figure 8a). Interestingly, the band gap feature of overlapping nanowires is con- 


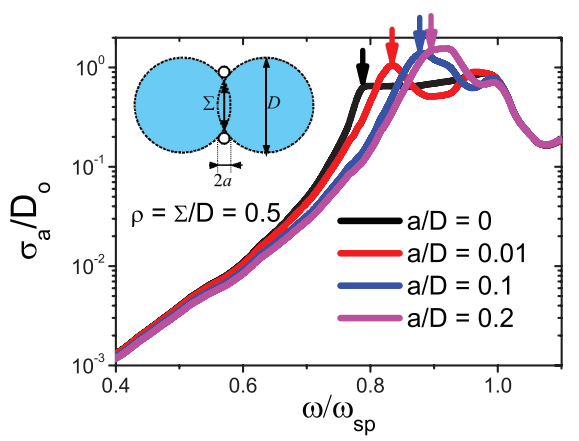

Figure 10. Normalized absorption cross section $\sigma_{\mathrm{a}}$ as a function of frequency and the radius $a$ of the two circles which are tangential to the overlapping cylinders' pair as shown in the inset. The cylinders pair of $10 \mathrm{~nm}$ diameter $D$ has a constant value of $\rho=0.5$ (blue). The arrows indicate the cutoff frequency of absorption for each device.

served even in presence of radiative losses. Figure 8a shows that the best light harvesting efficiency is obtained for cylinder diameter around $30 \mathrm{~nm}$. The radiative spectrum also exhibits a pronounced cutoff behavior for $D \leq 30 \mathrm{~nm}$ (see Figure 8b). On the contrary, for larger overlapping nanowires ( $D=100$ and $150 \mathrm{~nm}$ ), the radiative losses significantly increase below $\omega_{c}$ while the absorption losses are still negligible. Since surface plasmon modes are not excited in overlapping cylinders below $\omega_{c}$, most of the energy is scattered and not absorbed by the metallic nanowires.

Influence of Nanofabrication Imperfections on Light Harvesting. To be more realistic from an experimental point of view, the structure singularities of the overlapping nanoparti- cles are now slightly blunted in the numerical simulations. To that aim, two cylinders are inserted between the overlapping nanoparticles as shown in the inset of Figure 9. The triangle-shaped areas enclosed between the two nanowires, and these cylinders are filled with metal, which results in two smooth arcs that connect the two nanowires instead of two singular points. Figure 9 compares the absorption cross sections of these overlapping nanowires with the theoretical prediction. One can see that the bluntness of the structure singularities does not change significantly the cutoff absorption behavior for large overlap distances ( $\rho \geq 0.5$ ), which means that our theory initially developed for perfect overlapping nanowires is still effective in this case. The discrepancy between simulation and theory for the smaller overlap distance $(\rho=0.2$ ) is explained by the fact that the response of overlapping nanowires at low frequencies $\left(\omega<0.5 \omega_{\text {sp }}\right.$ ) relies on very sharp geometric features: the field is much more confined at low frequencies ${ }^{6}$ and any slight deviation from the initial geometry may prevent surface plasmons from being excited. Hence, if we want plasmonic devices efficient in the red part of the visible spectrum, strong constrains have to be imposed on the nanofabrication process.

The effect of the size of the nanofabrication imperfections is investigated in Figure 10. The absorption cross section of $10 \mathrm{~nm}$ diameter overlapping nanowires is shown for different radius $a$ of the inserted circles for $\rho=0.5$. As displayed by Figure 10 , the ab-
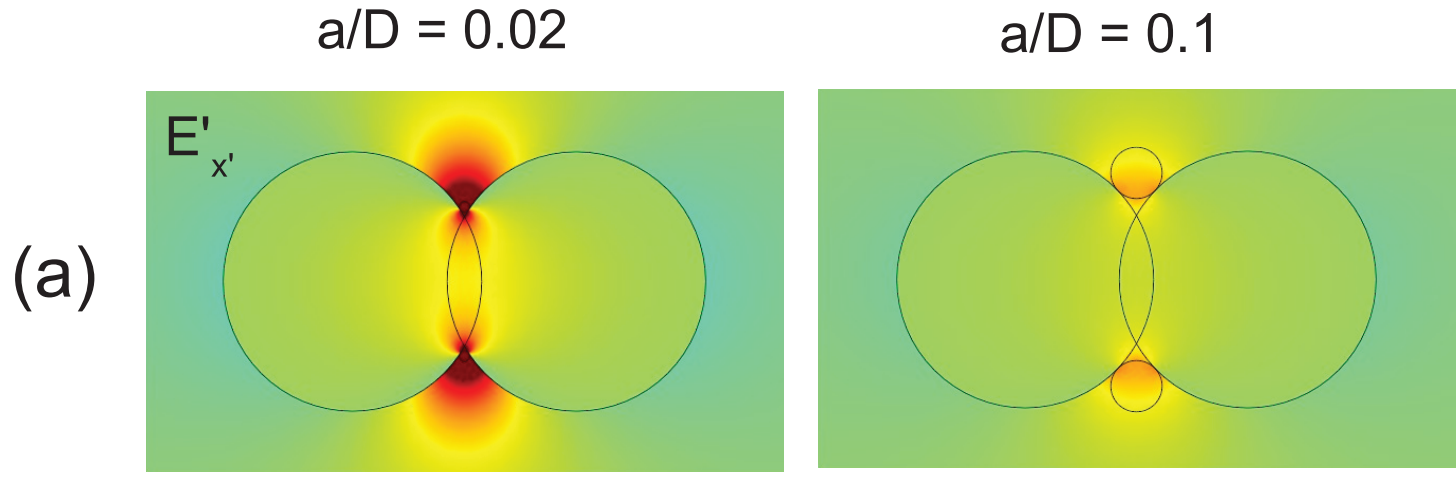
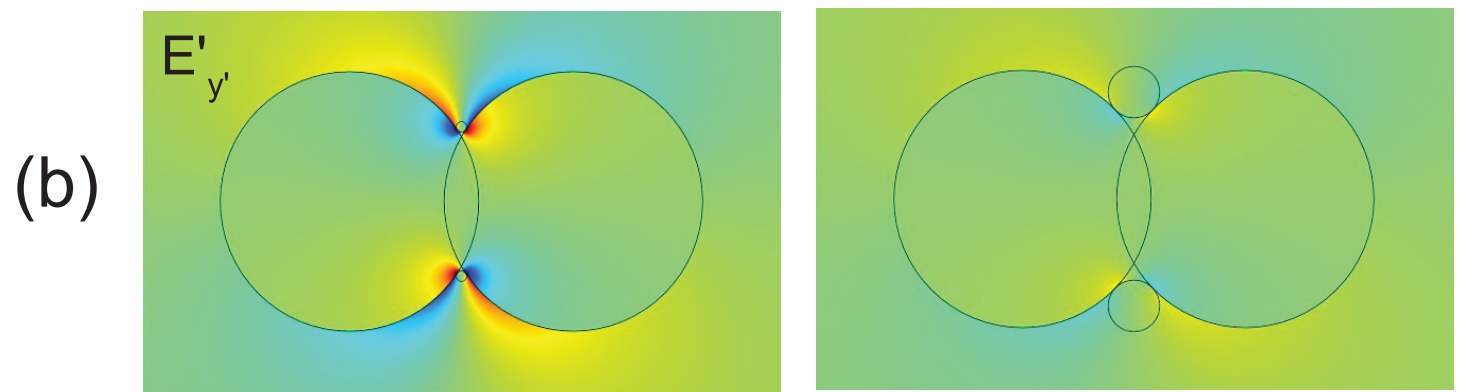

Figure 11. Amplitude of the real part of $E_{x^{\prime}}^{\prime}(a)$ and $E_{y^{\prime}}^{\prime}(b)$ normalized by the incoming field $E_{0}^{\prime}$ (polarized along $x^{\prime}$ ) at a frequency of $\omega=0.84 \omega_{\mathrm{sp}}$ for an overlapping nanowires' pair of $10 \mathrm{~nm}$ diameter $D$ and $\rho=0.5$ with singularities blunted by tangentially inserting two circles of 0.2 and $1.0 \mathrm{~nm}$ radii around the two crossing points of two cylinders as shown in the inset. The color scale is linear and restricted to [ -2020$]$ (corresponding to dark blue and dark red, respectively), but note that the field magnitude can be by far larger, especially near the overlap area. 
sorption cross section exhibits a robust cutoff response for a sample imperfection $a / D=0.01$, though two individual resonances emerge on both sides of the absorption band. Larger imperfections $(a / D=0.2)$ implies a blue-shift of the cutoff frequency as indicated by the arrows in the figure. The control of sample imperfections is thus important to obtain a stable performance in light harvesting within the absorption band of overlapping nanowires.

Influence of Nanofabrication Imperfections on Nanofocusing. As already pointed out previously above, the induced electric field may be divergent at the structure singularities for perfect overlapping nanowires. The bluntness of the singularities is expected to suppress this divergent feature. Figure 11 displays the electric field distributions for overlapping nanowires of $10 \mathrm{~nm}$ diameter $D$ and $\rho=$ 0.5 with a sample imperfection $a=D / 50$ (left column) and $a=D / 10$ (right column). The induced electric field is no longer divergent in absence of structure singularities and the field enhancement decreases with the size of the sample imperfection. However, both $E_{x^{\prime}}^{\prime}$ and $E_{y^{\prime}}$ still show a significant field enhancement. For instance, at a frequency of $0.84 \omega_{\text {sp }}$, a maximum field enhancement of 60 is obtained for a sample imperfection $a=$ $D / 50$ (see Figure 11a).

\section{CONCLUSION}

As a conclusion, conformal transformation theory has proven to be an elegant and powerful tool to predict analytically the optical response of overlapping nanowires in the quasi-static limit. Surface plasmon excitations in overlapping nanowires are shown to exhibit a lower bound cutoff frequency, which blue-shifts when the overlap distance increases. This cutoff response might open perspectives for the realization of plasmonic band gap filters. A divergent electric field distribution around structure singularities has also been predicted even in the presence of dissipation losses. Numerical simulations have confirmed the validity of our theory in the quasi-static limit. When structure dimensions become comparable to the wavelength, overlapping nanowires keep their band gap feature despite the radiative losses. At last, the unavoidable imperfections inherent in a nanofabrication process have been tested numerically. Slightly rounding off the structure singularities from overlapping nanowires does not significantly alter the cutoff absorption behavior for structures with large overlap distances but of course eliminate the divergent behavior of the electric field distribution. Nevertheless, a strong nanofocusing of light is still possible even when the structure singularities are slightly blunted.

\section{METHODS}

All numerical simulations have been performed by means of finite element method implemented in COMSOL Multiphysics software. Two dimensional simulations were performed within the harmonic propagation analysis mode in frequency domain. A plane wave with $x^{\prime}$-polarized electric field was introduced to the simulation area in $y^{\prime}$-direction (see the definition of coordinates in Figure $1 \mathrm{~b})$. The convergence of the numerical calculations with respect to the perfect-match-layer (PML) thickness, total simulation area, and mesh size has been checked. It was found that a PML thickness of $40 \mathrm{~nm}$ was sufficient to absorb the scattered fields at the simulation domain boundaries. A total simulation area above 4 $\mu \mathrm{m}^{2}$ was considered for the largest nanostructures studied in this work. The highly non-uniform and extremely fine mesh sides below $10^{-6} \mathrm{~nm}$ were used in order to model accurately the propagation of electromagnetic fields at the crossing points between two nanowires. Although optimal and rigorous conditions have been adopted in the simulations, the divergence problem cannot be completely eliminated for overlapping nanowires of small $\rho$ due to the fact that the electric field is actually divergent at these structure singularities, thereby leading to the slight oscillations in the absorption spectra as shown in Figure 7.

Acknowledgment. D.Y.L. and S.A.M. acknowledge support by the UK Engineering and Physical Sciences Research Council (EPSRC). A.A. and J.B.P. acknowledge support by the European Community project PHOME (Contract No. 213390). Y.L. acknowledges the Lee family scholarship for financial support.

\section{REFERENCES AND NOTES}

1. Kottmann, J. P.; Martin, O. J. F. Plasmon Resonant Coupling in Metallic Nanowires. Opt. Express 2001, 8, 655-663.

2. Prodan, E.; Radloff, C.; Halas, N. J.; Nordlander, P. A Hybridization Model for the Plasmon Response of Complex Nanostructures. Science 2003, 302, 419.

3. Atay, T.; Song, J.-H.; Nurmikko, V. Strongly Interacting Plasmon Nanoparticle Pairs: From Dipole-Dipole Interaction to Conductively Coupled Regime. Nano Lett. 2004, 4, 1627-1631.

4. Nordlander, P.; Oubre, C.; Prodan, E.; Li, K.; Stockman, M. I. Plasmon Hybridization in Nanoparticle Dimers. Nano Lett. 2004, 4, 899-903.

5. Romero, I.; Aizpurua, J.; Bryant, G. W.; García de Abajo, F. J. Plasmons in Nearly Touching Metallic Nanoparticles. Opt. Express 2006, 14, 9988-9999.

6. Aubry, A.; Lei, D. Y.; Fernandez-Dominguez, A. I.; Sonnefraud, Y.; Maier, S. A.; Pendry, J. B. Plasmonic Light Harvesting Devices over the Whole Visible Spectrum. Nano Lett. 2010, 10, 2574-2579.

7. Lei, D. Y.; Aubry, A.; Maier, S. A.; Pendry, J. B. Broadband Nano-Focusing of Light Using Kissing Nanowires. New J. Phys. 2010, 12, 093030.

8. Aubry, A.; Lei, D. Y.; Maier, S. A.; Pendry, J. B. Broadband Plasmonic Device Concentrating the Energy at the Nanoscale: The Crescent-Shaped Cylinder. Phys. Rev. B 2010, 82, 125430.

9. Luo, Y.; Pendry, J. B.; Aubry, A. Surface Plasmons and Singularities. Nano Lett. 2010, 10, 4186-4191.

10. Aubry, A.; Lei, D. Y.; Maier, S. A.; Pendry, J. B. Conformal Transformation Applied to Plasmonics beyond the QuasiStatic Limit. Phys. Rev. B 2010, 82, 205109.

11. Aubry, A.; Lei, D. Y.; Maier, S. A.; Pendry, J. B. Interaction between Plasmonic Nanoparticles Revisited with Transformation Optics. Phys. Rev. Lett. 2010, 105, 233901.

12. Nie, S.; Emory, S. R. Probing Single Molecules and Single Nanoparticles by Surface-Enhanced Raman Scattering. Science 1997, 275, 1102.

13. Moskovits, M. Surface-Enhanced Spectroscopy. Rev. Mod. Phys. 1985, 57, 783-826.

14. Kim, S.; Jin, J.; Kim, Y. J.; Park, I. Y.; Kim, Y.; Kim, S. W. HighHarmonic Generation by Resonant Plasmon Field Enhancement. Nature 2008, 453, 757. 
15. Bora, M.; Fasenfest, B. J.; Behymer, E. M.; Chang, A. S-P; Nguyen, H. T.; Britten, J. A.; Larson, C. C.; Chan, J. W.; Miles, R. R.; Bond, T. C. Plasmon Resonant Cavities in Vertical Nanowire Arrays. Nano Lett. 2010, 10, 2832.

16. McPhedran, R. C.; Perrins, W. T. Electrostatic and Optical Resonances of Cylinder Pairs. Appl. Phys. 1981, 24, 311-318.

17. McPhedran, R. C.; Milton, G. W. Transport Properties of Touching Cylinder Pairs of the Square Array of Touching Cylinders. Proc. R. Soc. A 1987, 411, 313-326.

18. Radchik, A. V.; Smith, G. B.; Reuben, A. J. Quasistatic Optical Response of Separate, Touching, and Intersecting Cylinder pairs. Phys. Rev. B 1992, 46, 6115-6125.

19. Johnson, P. B.; Christy, R. W. Optical Constants of the Noble Metals. Phys. Rev. B 1972, 6, 4370-4379.

20. Bohren, C. H.; Huffman, D. R. Absorption and Scattering of Light by Small Particles; John Wiley \& Sons, Inc.: New York, 1983.

21. Kreibig, U.; Vollmer, M. Optical Properties of Metal Clusters; Springer: Berlin, 1995.

22. Palik, E. D. Handbook of Optical Constants of Solids; Academic: New York, 1991; Vol. II. 